\title{
Significance of tumor protein p53 mutation in cellular process and drug selection in brain lower grade (WHO grades II and III) glioma
}

\author{
Chang-feng Guo ${ }^{1}$, Yugang Zhuang ${ }^{1}$, Yuanzhuo Chen ${ }^{1}$, Sheng Chen ${ }^{1}$, Hu Peng ${ }^{1}$ \& Shuqin \\ Zhou*,1 (iD) \\ ${ }^{1}$ Department of Emergency, Shanghai Tenth People's Hospital, Tongji University, School Of Medicine, Shanghai, 200072, China \\ *Author for correspondence: zhoushuqintju@163.com
}

\begin{abstract}
Aim: Tumor protein p53 (TP53) mutant is one of the most frequently mutated genes in glioma. Results: The Cancer Genome Atlas data has shown that TP53 mutation is present in $49 \%$ of lower grade (World Health Organization [WHO] grades II and III) glioma patients. Data from The Genomics of Drug Sensitivity in Cancer database showed that three drugs: (5Z)-7-oxozeaenol, dabrafenib and nutlin-3a (-), have shown more resistance in patients with TP53 mutation. We identified 1100 differentially expressed genes. Functional enrichment analysis showed that the differentially expressed genes are mainly concentrated in the transport of ionic and cancer-related pathways. The top ten hub genes were identified and an outcome analysis revealed the most critical genes related to prognosis. Conclusion: Our results identified the key genes and pathways that might provide the basic proof to improve individualized treatment in patients with glioma.
\end{abstract}

First draft submitted: 4 June 2020; Accepted for publication: 1 July 2020; Published online: 15 July 2020

Keywords: bioinformatics analysis $\bullet$ brain lower grade glioma $\bullet$ RNA sequencing $\bullet$ TCGA $\bullet$ TP53 mutation

Glial cells are the main cells which support the brain's nerve cells and keep them healthy. As we all know, glioma is a cancer that develops in the brain's glial cells [1]. Glioma is the most common CNS tumor and is prevalent in about half of all intracranial primary tumors, and all neuroepithelial-derived tumors. It refers to all tumors originating from various types of glial cells. In 2016, the World Health Organization (WHO) established a new concept of molecular diagnosis of such tumors, which were divided into oligodendroglioma with a $I D H$ mutation, as well as a $1 \mathrm{p} / 19 \mathrm{q}$ codeletion, diffuse astrocytoma with a $I D H$ mutation, anaplastic oligodendroglioma $(I D H$-mutated and 1p/19q codeletion), $I D H$-mutated glioblastoma, $I D H$-mutated anaplastic astrocytoma, $I D H$ wild-type glioblastoma, diffuse midline glioma with H3K27M mutation and so on. Moreover, tumors are classified clinically as grade I, II, III or IV according to the standards set by the WHO [2]. Based on this classification, low- and intermediate-grade gliomas (WHO II and III) are also called lower-grade gliomas (LRGGs), which have highly variable clinical behaviors that are not adequately predicted on the basis of histologic class. In this study, we focus on these LRGGs [3-5]. Despite the grade, glioma will compress normal brain tissue and cause pressure and space related disabilities or fatal consequences as it grows in size. In 2010, more than 22,000 Americans were estimated to be diagnosed, and an estimated 13,140 people have died of complications related to brain and other neurological cancers [6].

Nowadays, the choice of individualized therapy is currently based on the results of molecular pathological analysis and individualized drug testing. These tests can help doctors choose more suitable chemotherapeutic agents based on the different characteristics of patients' tumor cells for personalized treatment, which could significantly delay disease progression and improve prognosis. In summary, the ability to determine tumor characteristics of glioma patients can provide a theoretical basis for better-individualized treatment.

TP53 is a well-known tumor suppressor that plays a central role in many tumors [7]. It can play multiple roles in complex cellular processes by regulating the expression of target genes [8]. A mutation in TP53 is found in multiple cancer types, including glioma [9]. Previous results indicated that almost 50\% of the glioma samples had a TP53 mutation [10]. A TP53 mutation can promote the disease program and affect treatment strategies [11,12]. Many previous studies have shown that mutant TP53 can accelerate cell proliferation and promote cancer cell

Future Medicine 
metastasis [13]. Since the TP53 mutation is one of the most commonly detected mutations in glioma patients, we plan to explore whether we can define glioma patients with a TP53 mutation as a subgroup, thus helping identify some novel personalized treatment methods.

In this study, we used the data in the Genomics of Drug Sensitivity in Cancer (GDSC) database to assess the significance of TP53 mutations in the selection of chemotherapy drugs for glioma. In addition, the RNA sequencing (RNA-seq) data of brain LRGG was downloaded and analyzed to explore the role of TP53 mutation in disease progression, thereby providing novel personalized treatment options.

\section{Materials \& methods}

RNA-seq data

We downloaded brain LRGG RNA-seq data directly from The Cancer Genome Atlas (TCGA) database. Then we obtained the corresponding clinical information from the cBioPortal for the Cancer Genomics website (Brain LRGG, TCGA and the PanCancer Atlas) [14].

\section{Analysis of the GDSC database}

The GDSC project is mainly used for cancer molecular treatment and mutation exploration [15]. Public online platforms have been developed to help researchers analyze and download relevant data [16]. In this study, we searched for the compounds with selectivity for TP53 mutation at first. Then we searched for tumor chemotherapy drugs specific to LRGG cells and downloaded the volcano plots and scatter plots directly from the GDSC online platform.

\section{Gene set enrichment analysis}

We analyzed the genetic differences of biological annotations and pathways in gene set enrichment analysis (GSEA) to help us further understand the effect of TP53 mutations on the biological function of patients with LRGG. We downloaded the GSEA 4.0.3 software directly from the Broad Institute website (https:/www.gsea-msigdb.org/gs ea/index.jsp). Then we imported the data matrix in the software and the number of permutations was set at 100 . H.all.v7.1.symbols.gmt was chosen as the gene sets database. Enrichment results satisfying the p-value $<0.05$ with a false discovery rate $<0.25$ were considered statistically significant.

\section{Identification of differentially expressed genes}

We divided the 508 LRGG patients from TCGA into a TP53 mutant group and a TP53 wild-type group according to whether TP53 was mutated. We used EdgeR to find out differentially expressed genes (DEGs) between TP53 mutation and wild-type groups $[17,18]$. Genes with fold change $\geq 2$ or $\leq 0.5$ and the $p$-value $<0.05$ were identified as DEGs.

\section{Gene ontology \& pathway enrichment analysis of DEGs}

In this study, the database for annotation, visualization and integrated discovery (DAVID; https://david.ncifcrf.go v/.) was employed to deal with gene functional analysis [19]. In this section, we mainly focused on gene ontology (GO) and Kyoto encyclopedia of genes and genomes (KEGG) analysis. All the DEGs were uploaded to the DAVID website for enrichment analysis, while specifying a $\mathrm{p}<0.05$ for statistical significance.

\section{Analyses of protein-protein interaction networks \& module}

A search tool for the retrieval of interacting genes (STRING) database provides a critical assessment and integration of protein-protein interaction (PPI) for researchers to analyze protein interaction [20]. We used this database in the analysis of PPI. To evaluate the interaction among all the DEGs we have identified, we submitted all the DEGs to the STRING website. Then, only experimentally validated interactions (total score $>0.4$ ) were selected as meaningful. The PPI network was then used for module screening by molecular complex detection in Cytoscape (scores $>3$, nodes $>4$ ) [21]. The hub genes, which are highly interconnected with the nodes in the module, have shown important functions. Nodes with a connection degree $>10$ were selected as hub genes.

\section{Statistical analyses}

We used student $t$-tests to compare gene mRNA expression data in TP53 mutants and wild-type LRGG tissues. As for the prognosis analysis, expression levels of the selected hub genes were divided into high- or low-expression levels according to the median value at the cut-off point. Then, the Kaplan-Meier method and log-rank test using 


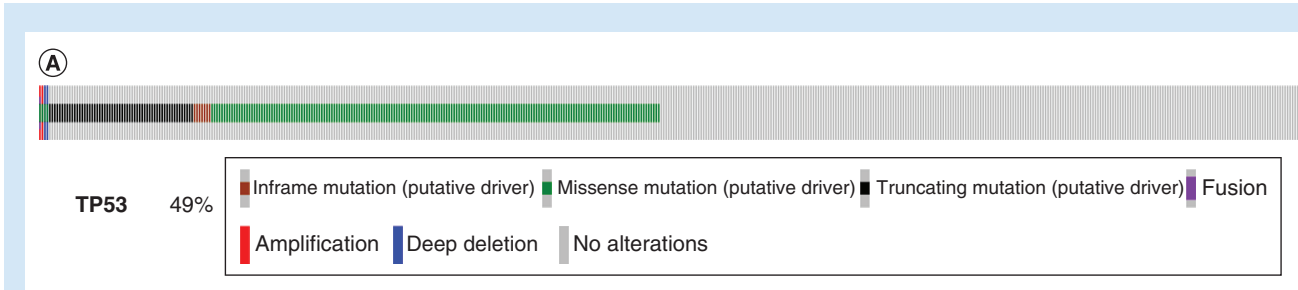

\begin{tabular}{|c|c|}
\hline Mutation type & Incidence \\
\hline $\begin{array}{c}\text { Inframe mutation } \\
\text { (putative driver) }\end{array}$ & $1.38 \%$ \\
\hline $\begin{array}{c}\text { Missense mutation } \\
\text { (putative driver) }\end{array}$ & $36.29 \%$ \\
\hline $\begin{array}{c}\text { Truncating mutation } \\
\text { (putative driver) }\end{array}$ & $11.44 \%$ \\
\hline
\end{tabular}

(B)

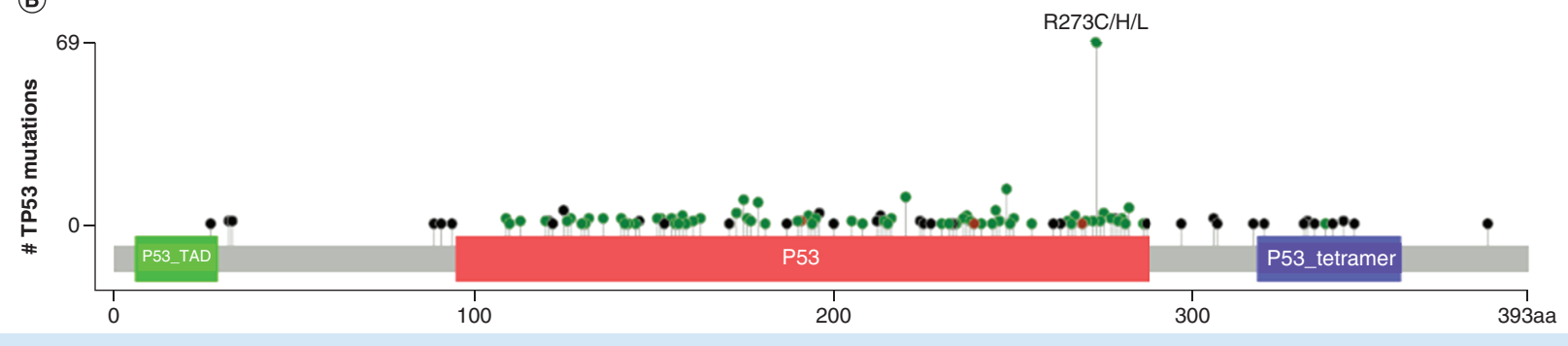

Figure 1. Mutation frequency and types of tumor protein p53 in lower grade glioma from The Cancer Genome Atlas database. (A) Mutation frequency and mutation types of TP53 in lower grade glioma. (B) Figure showed where missense mutations and truncating mutations locate on the protein.

TAD: Transactivation motif; Tetramer: Tetramerization motif.

Graphpad were used to calculate clinical prognosis. All statistical analyses were performed using Graphpad. p < 0.05 was considered statistically significant.

\section{Results}

\section{TP53 mutation is highly detected in LRGG}

We downloaded tumor tissue gene expression data and completed clinical follow-up data of 508 patients with LRGG from the TCGA database. Of all patients, 250 patients had TP53 mutation, accounting for approximately $49 \%$ of the total (Figure 1A). In the cases of TP53 mutation, the types of mutations include amplification, truncation, deep deletion, in-frame mutations and missense mutations that span the entire gene (Figure 1B).

\section{TP53 mutation does not influence LRGG prognosis}

Next, we explored the impact of TP53 mutations on prognosis and treatment. We first investigated TP53 mRNA expression in TP53 wild-type and mutated groups. Our results showed that there was no significant difference in TP53 expression levels between the two groups (Figure 2A). Additionally, we identified that the TP53 mutation was found to have no effect on disease prognosis (Figure 2B).

\section{LRGG cells with TP53 mutation are resistant to (5Z)-7-oxozeaenol, dabrafenib \& nutlin-3a (-)}

The sensitivity and resistance to chemotherapeutic drugs determines the outcome of therapy. We further investigated whether the TP53 mutation has any impact on the increase or decrease in the sensitivity or resistance of chemotherapeutic drugs in LRGG. We queried the GDSC database to determine whether the TP53 mutant cells had specific therapeutic drugs or compounds. The results suggested that four drugs: (5Z)-7-oxozeaenol (OZ), bleomycin, dabrafenib and nutlin-3a (-) were both resistant in cancer cells with a TP53 mutation (Figure 3). Furthermore, we explored the drug resistance or sensitivity in LRGG cells, and found that LRGG cells that harbor a TP53 mutation, were significantly resistance to only (5Z)-7-OZ, dabrafenib and nutlin-3a (-) (Figure 4). We discovered that for LRGG patients with TP53 mutation, (5Z)-7-OZ, dabrafenib and nutlin-3a (-) may not be therapeutically suitable compounds.

\section{Results of GSEA}

Our results indicated that the TP53 mutation plays a critical role in drug selection for LRGG. We then analyzed the effects of TP53 mutations on cellular processes. We first analyzed the GSEA method to deal with biological 


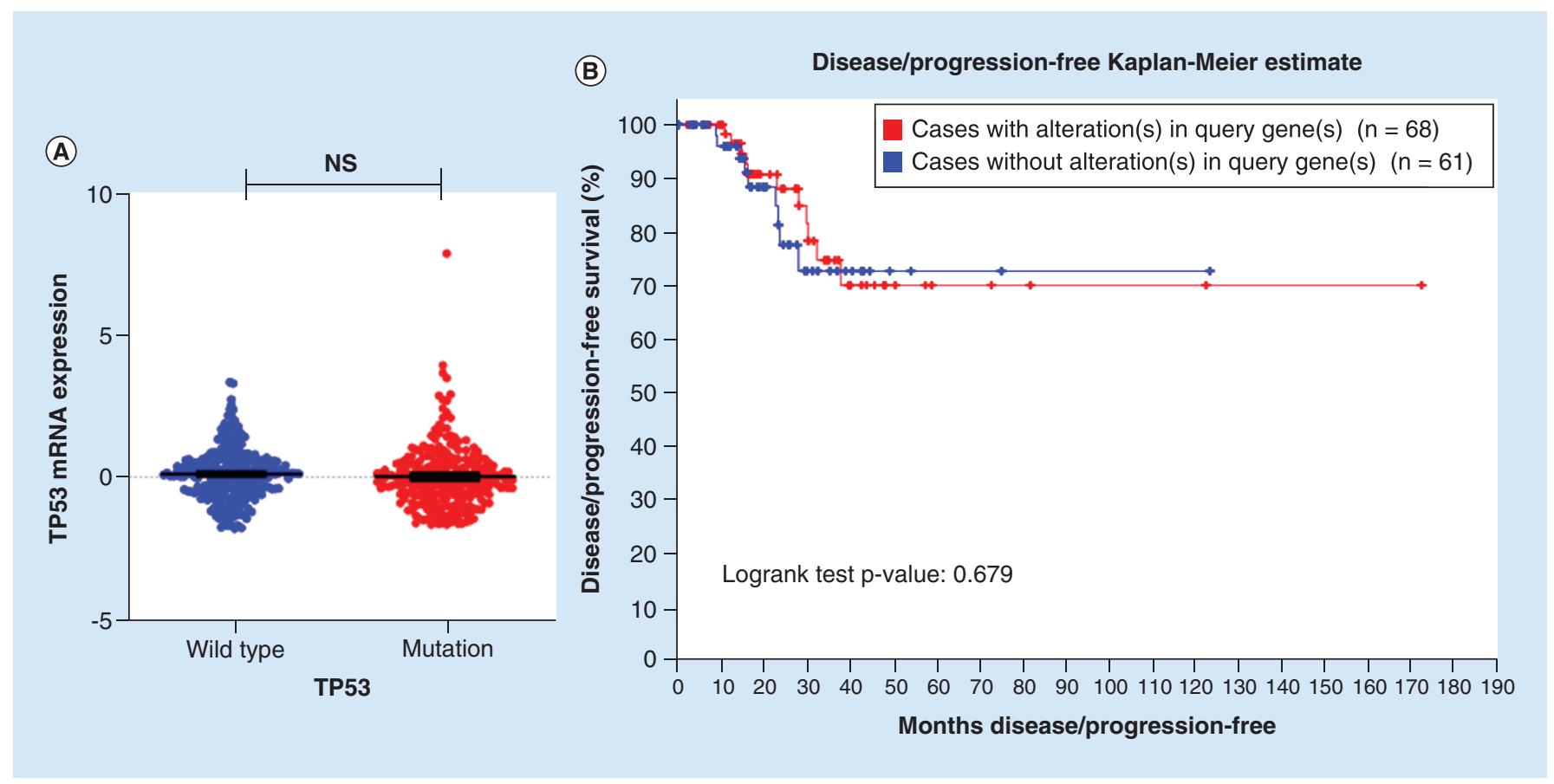

Figure 2. Mutation of tumor protein p53 and lower grade glioma prognosis. (A) Correlation between TP53 mutation and mRNA expression. (B) Kaplan-Meier survival curves for lower grade glioma patients stratified by TP53 mutation.

Log-rank test was used for analysis and a p-value $<0.05$ for statistical significance.

NS: Not significant.

functional gene sets. The results in Figure 5 indicated that $T G B-\beta$ signaling, $N O T C H$ signaling and mitotic spindles were significantly enriched, which suggests that the TP53 mutation may influence LRGG progression and drug selection, by influencing the aforementioned pathways and cell division processes.

\section{Identification of DEGs}

Next, we identified DEGs in gene expression data to find pathways and genes related to a TP53 mutation. We divided the 508 LRGG patients into a TP53 mutant group and a TP53 wild-type group according to whether TP53 was mutated. According to the results of analysis, we identified a total of 1100 DEGs, of which 479 were raised and 621 were downregulated. The DEG volcano map is shown in Figure 6A.

\section{GO \& KEGG analysis of DEGs}

We then uploaded the above 1100 DEGs that we collected and performed functional enrichment analysis through DAVID. The results of the GO analysis (Figure 6B) showed that potassium ion transmembrane transport, nervous system development, chloride transport, regulation of membrane potential, cell-cell signaling, ion transmembrane transport, transport, visual perception, regulation of ion transmembrane transport, axon guidance, cognition, cell development, regulation of DNA biosynthetic process, extracellular matrix organization and response to wounding were significantly enriched.

The KEGG pathway analysis showed a significant enrichment in neuroactive ligand-receptor interaction, cAMP signaling pathway, aldosterone synthesis and secretion, insulin secretion, calcium signaling pathway, salivary secretion, glycosphingolipid biosynthesis - lacto and neolacto series, morphine addiction, thyroid hormone synthesis and ovarian steroidogenesis (Figure 6C).

Module screening from the PPI network \& the identification of prognosis related critical hub genes In order to study the interactions of DEGs and the hub genes, we subsequently conducted research through the STRING database. Molecular complex detection helped us identify the modules in the PPI network. To find out important networks, we showed the top three significant modules (Figures 6D-F). To further find out critical genes that might contribute to the change of drug sensitivity, we did a hub gene analysis. Hub genes were selected with 


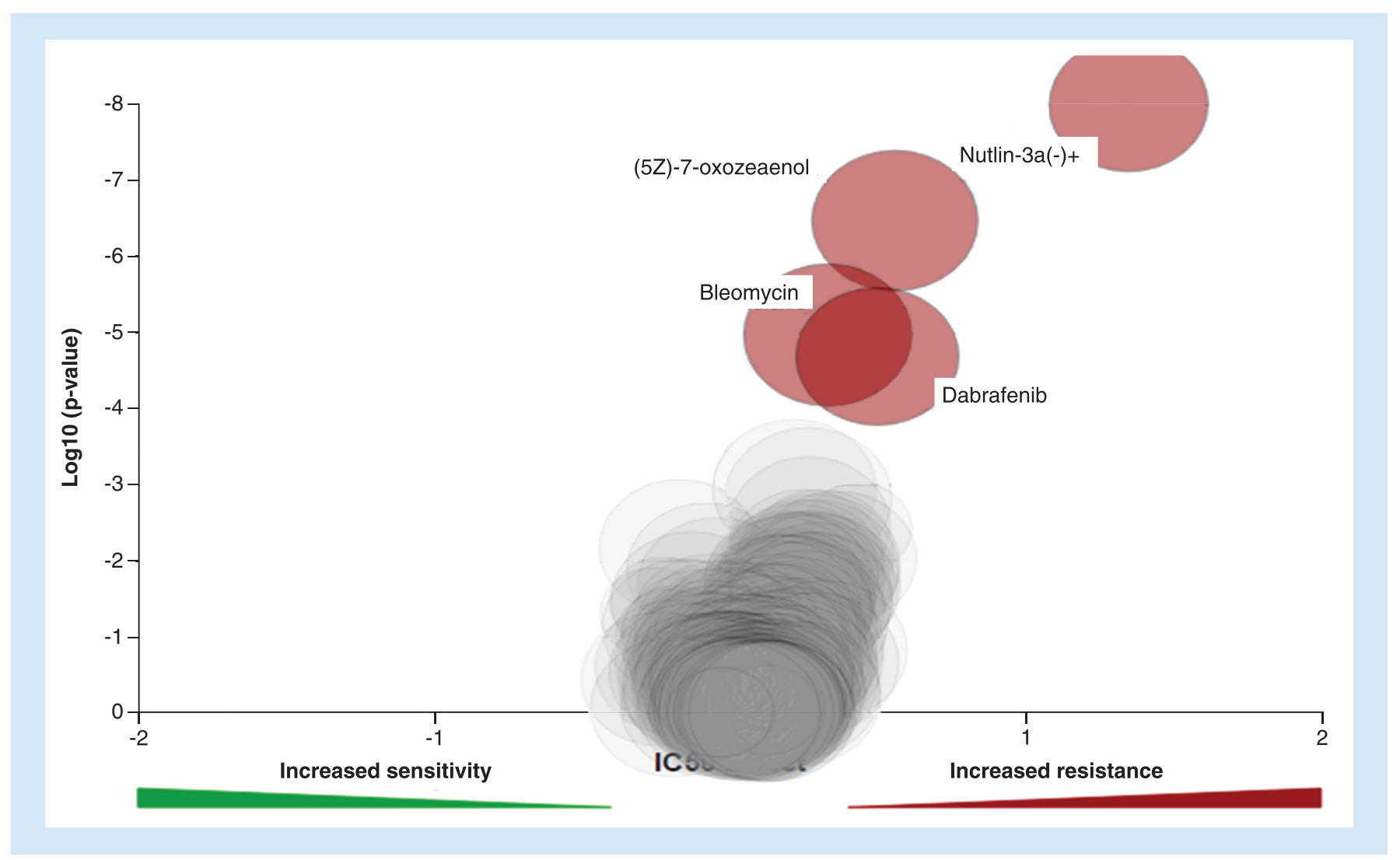

Figure 3. Volcano plotting showed that cancer cells with tumor protein p53 mutation were significantly resistant to (5Z)-7-oxozeaenol, bleomycin, dabrafenib and nutlin-3a (-).

a connection degree of $>10$. Then we ranked the hub genes according to this degree and the ten genes with the highest degree are regarded as the most critical hub genes, which included: SNAP25, GRM5, SNCB, HRH3, SYT4, GABRG2, ATP2B3, ADCY8, PRKACG and CPLX2. SNAP25 had the highest degree among all the hub genes in the list with a degree of 32 .

Then we analyzed whether the hub genes identified above have any relation to disease prognosis. Expression levels of the selected hub genes were divided into high or low expression levels according to the median value. Then, we used the Kaplan-Meier method with log-rank test to calculate clinical prognosis. The expression levels of $C P L X 2$, $S N A P 25, S N C B$ and $A T P 2 B 3$ were significantly correlated with the disease-free survival (DFS) rate of patients (Figures 7A-D). We also found that the expression levels of SNAP25, SNCB, SYT4, ATP2B3, CPLX2, GABRG2 and $H R H 3$ were significantly correlated with the overall survival (OS) rate of patients (Figures 7E-K). Other genes showed no influence on disease prognosis (Supplementary Figure 1). These results indicated the importance and significance of such genes in identifying and calculating disease prognosis, which might have potential research objectives in the future.

\section{Discussion}

TP53 contains multiple regulatory regions, which could complete transcriptional activation, DNA binding and other biological functions. TP53 acts as a tumor suppressor in many types of tumors. So far, TP53 mutations have been found to be ubiquitous in all cancer types [22]. TP53 can respond to a variety of cellular stresses, regulate the expression of target genes, and induce cell cycle arrest, aging, apoptosis, DNA repair, or metabolic processes [23-25]. A TP53 mutation is common in many human cancers, including glioma.

In this study, we evaluated the significance of a TP53 mutation in drug selection and disease progression in LRGG, providing a basis for individualized treatment. We found that data from GDSC showed preliminary evidence that (5Z)-7-OZ, dabrafenib and nutlin-3a (-) exhibit resistance to LRGG cells with a TP53 mutation. Such results may provide evidence that use of the potential drugs above, might result in ineffective medication in clinical practice. 

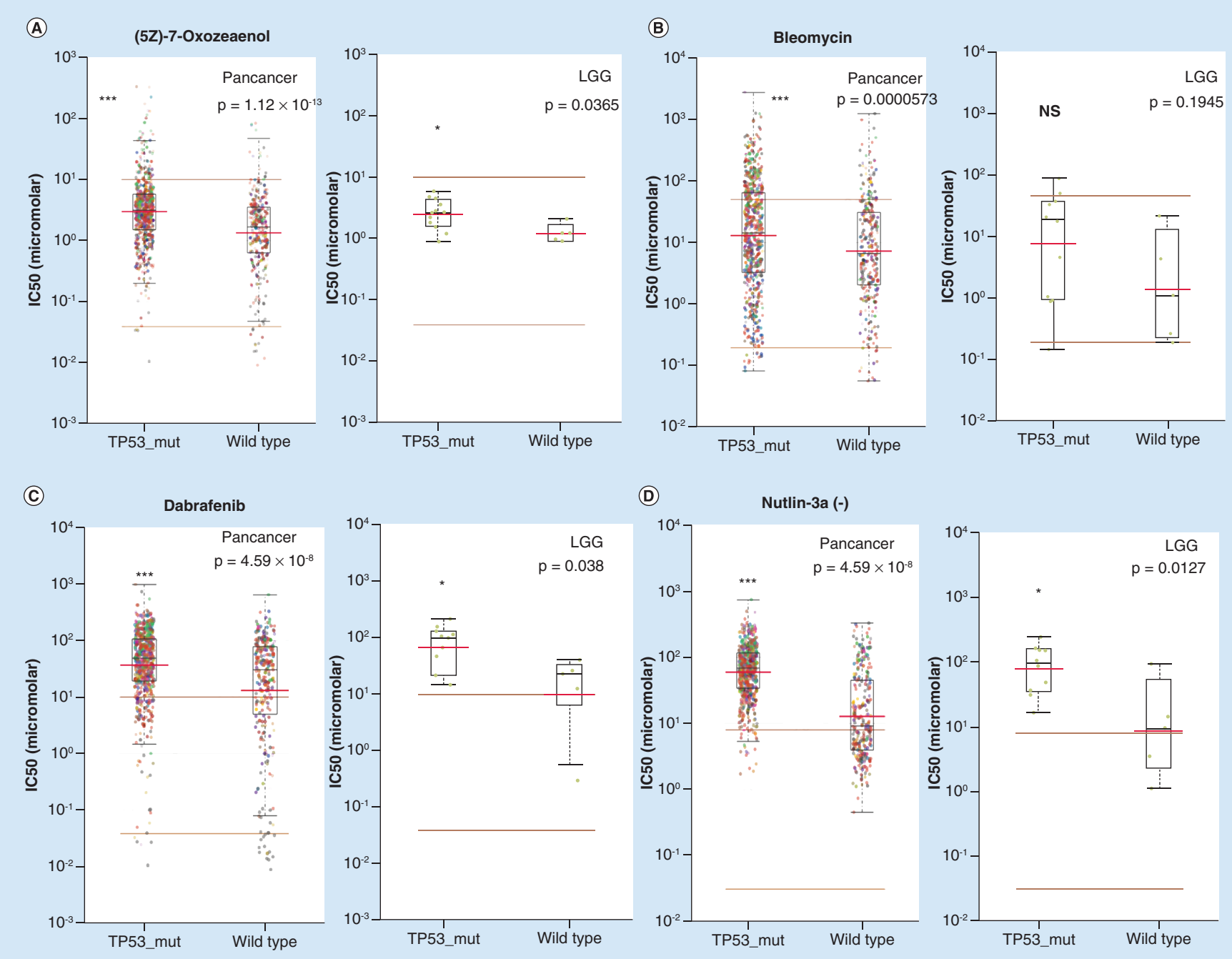

Figure 4. Mutation of tumor protein p53 influences drug selection of lower grade glioma. (A-D) Reproduction of the GDSC database showed that lower grade glioma cells with TP53 mutation, but not cancer of other types, was significantly resistant to (5Z)-7-oxozeaenol, dabrafenib and nutlin-3a (-), but not bleomycin.

GDSC: Genomics of drug sensitivity in cancer; $I_{50}$ : Half maximal inhibitory concentration; LGG: Lower grade glioma.

5Z-7-OZ is a TAK1 inhibitor that could attenuate early brain injury after experimental subarachnoid hemorrhage and has been found to be effective in various diseases in CNS [26-28]. Dabrafenib, an inhibitor of mutated BRAF, has clinical activity in patients with BRAF V600-mutated metastatic melanoma and is a promising new therapeutic drug in treating glioma [29-32]. As for nutlin-3a (-), previous studies have recently shown that in glioma cells, activation of TP53 by nutlin-3a decreases the expression level of $\alpha 5$ integrin to inhibit cell malignance [33]. Our findings may provide new evidence for finding better and more effective individualized treatment strategies.

In order to study mechanisms related to TP53 mutation, we downloaded and analyzed the RNA-seq data of LRGG to identify some critical signal pathways and hub genes. The results of the GSEA analysis showed that enriched gene sets were mainly associated with TGF- $\beta$ signaling, NOTCH signaling and mitotic spindles, which are all important contributors to LRGG initiation and development [3,34]. We then identified genes with differential expression and performed functional enrichment analysis of these genes. The results showed that a total of 1100 DEGs were found with different TP53 mutation states. The differential genes of patients with TP53-mutated LRGG are closely related to various cellular processes. Among all of the programs, we found that programs related to ion transport were enriched significantly. As we all know, ion transport has been shown to be critical in LRGG 

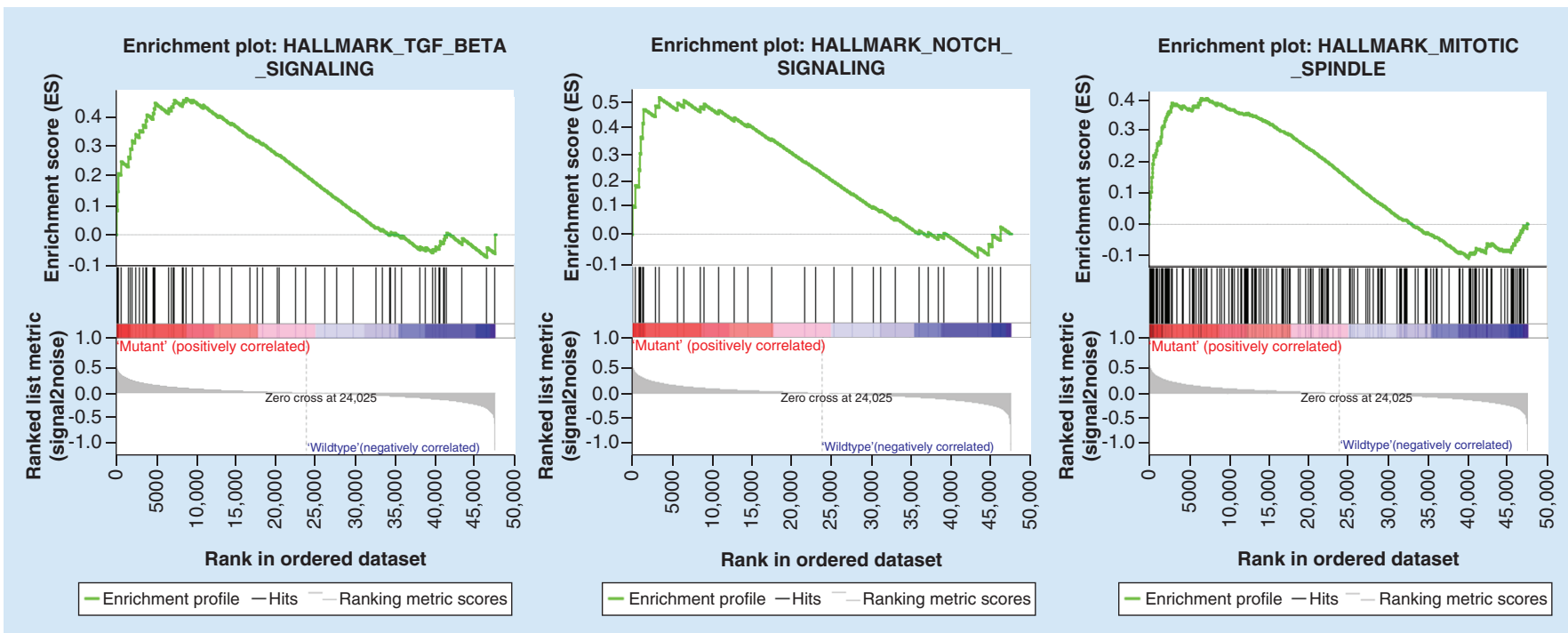

Figure 5. Gene set enrichment analysis results showed primary biological functional gene sets enriched in tumor protein p53 mutation in lower grade glioma patients, including TGF- $\beta$ pathway, NOTCH signaling pathway and mitotic spindles.

development and treatment [35,36]. Different transportation efficiency may influence the drug transmembrane and transpiration, which might provide possible mechanisms of drug resistance.

In particular, the KEGG pathway analysis showed significant pathways that might contribute to disease progression. A previous study showed that inactivation of neuroactive ligand-receptor interaction pathway could decrease the survival of glioblastoma patients drastically [37]. LRGG with TP53 mutation showed no significant difference with other patients, but whether the enrichment of the neuroactive ligand-receptor interaction pathway might contribute to drug resistance, could be an intriguing issue for further study. As important signaling pathways in cells, cAMP and calcium signaling pathways mediate many important signaling pathways, thus playing important roles in the progression of brain cancer [38]. These signal pathways might play important roles as targets for investigating drug resistance. In the present study, we also found that the synthesis and secretion of some metabolites and hormones, such as aldosterone, insulin, steroidogenesis and glycosphingolipid, were enriched in TP53-mutated LRGG patients. Although the details of them are still unclear and could involve some endocrine changes caused by the tumor, these findings provide interesting ideas for further study of the pathogenesis and drug selection for such patients.

In the PPI analysis, we found the top ten genes with the highest degree of interaction and defined them as hub genes. SNAP25 was demonstrated to be the top one. SNAP25 is a presynaptic plasma membrane protein regulating the release of neurotransmitters, and has been shown to have a potential biologic function in glioma [39]. We also revealed the significance of SNAP25 in disease prognosis of DFS or OS. Our results implied the potential role of SNAP25 and indicated that SNAP25 may be a promising therapeutic target in glioma [40]. Our prognosis analysis results also pointed out that several other central genes, including $S N C B, S Y T 4, A T P 2 B 3, C P L X 2, G A B R G 2$ and $H R H 3$, are closely related to the prognosis of the disease. Among them, CPLX2, SNCB and ATP2B3 are related to the DFS and OS rate, prompting their importance for disease progression.

Our study contained one limitation. In the present study, we only investigated the roles of TP53 mutation in LRGG for disease prognosis and drug sensitivity and found critical pathways and hub genes. The mechanism and validation of the TP53 mutation in this disease still needs further research in molecular biologic and clinical experiments. Moreover, it will be interesting to see how patients with TP53 mutations respond to gold standard therapy (such as temozolomide) in terms of OS and progression-free survival, which we will investigate in future work. 


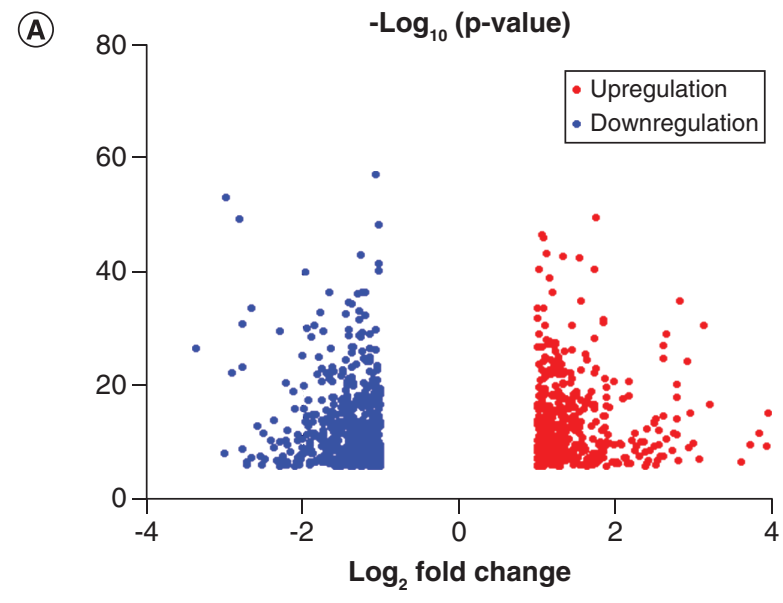

(C)

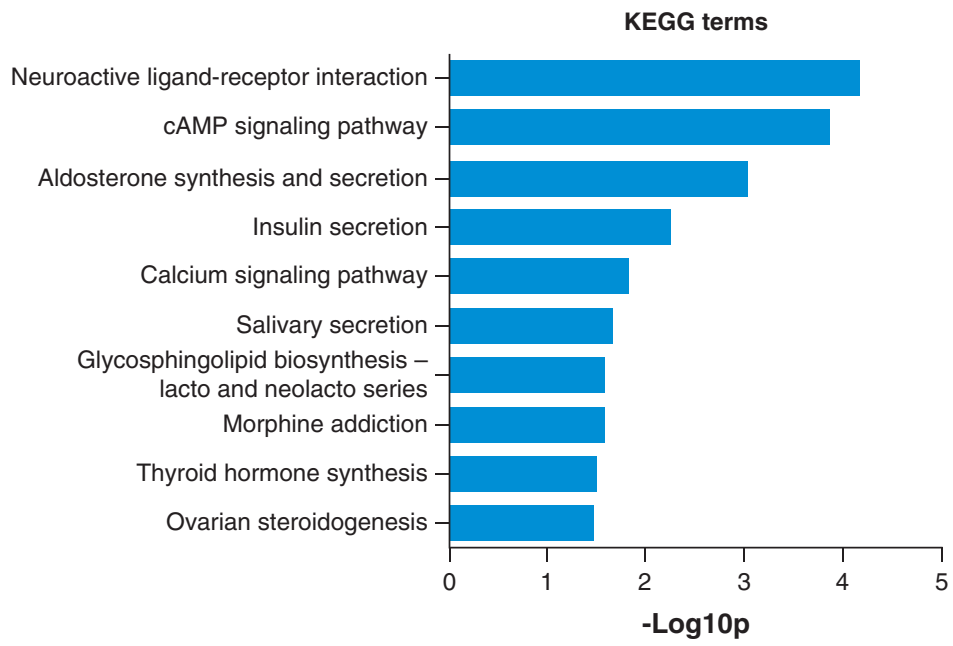

(ㄷ)

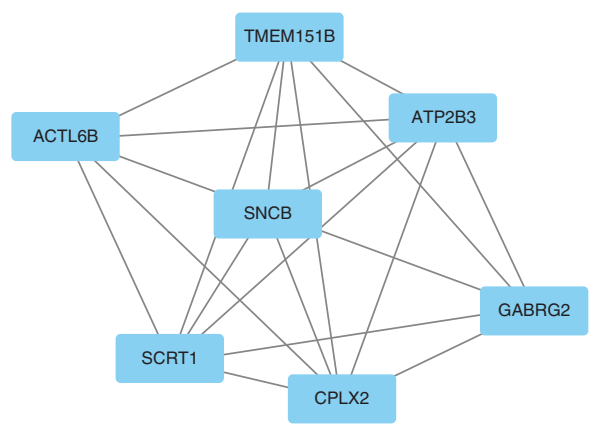

(B)

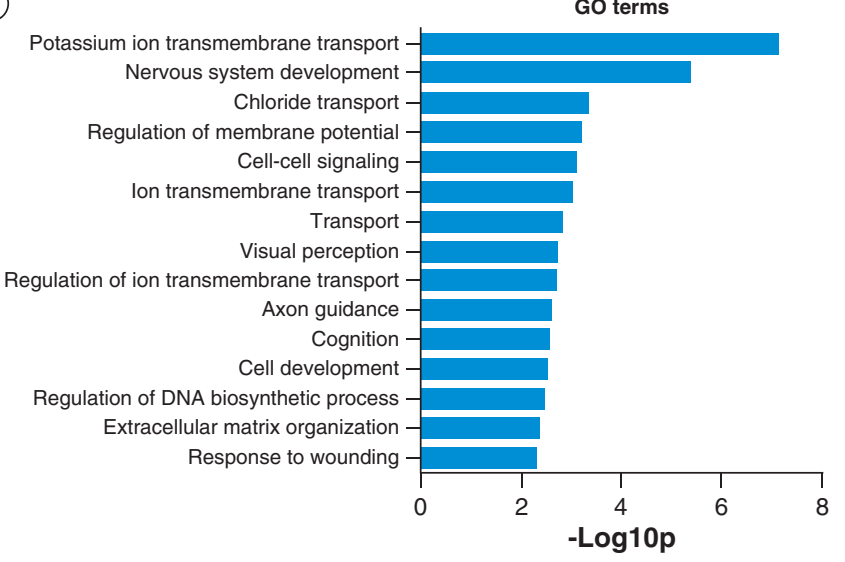

(D)

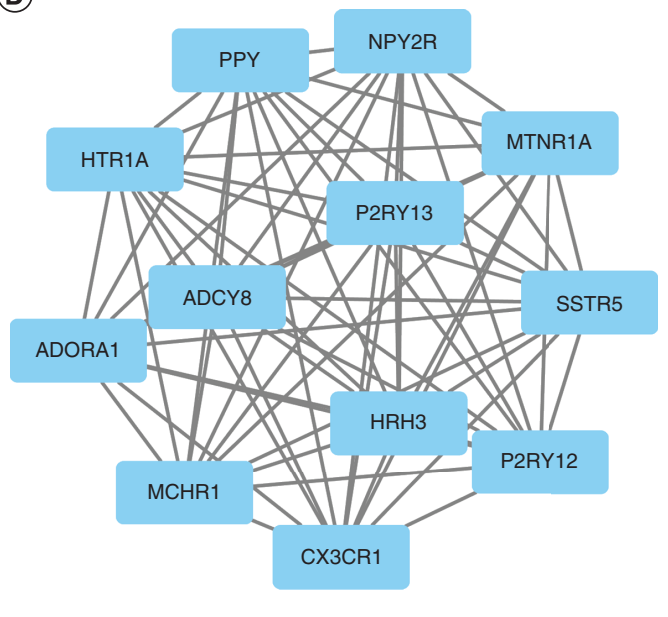

Figure 6. The database for annotation, visualization and integrated discovery enrichment results of differentially expressed genes. (A) Volcano plot for differentially expressed genes. (B) The GO enrichment terms of differentially expressed genes. (C) The KEGG pathway analysis of differentially expressed genes. (D) PPI network of module top one. (E) PPI network of module top two. (F) PPI network of module top three.

GO: Gene ontology; KEGG: Kyoto encyclopedia of genes and genomes; PPI: Protein-protein interaction. 

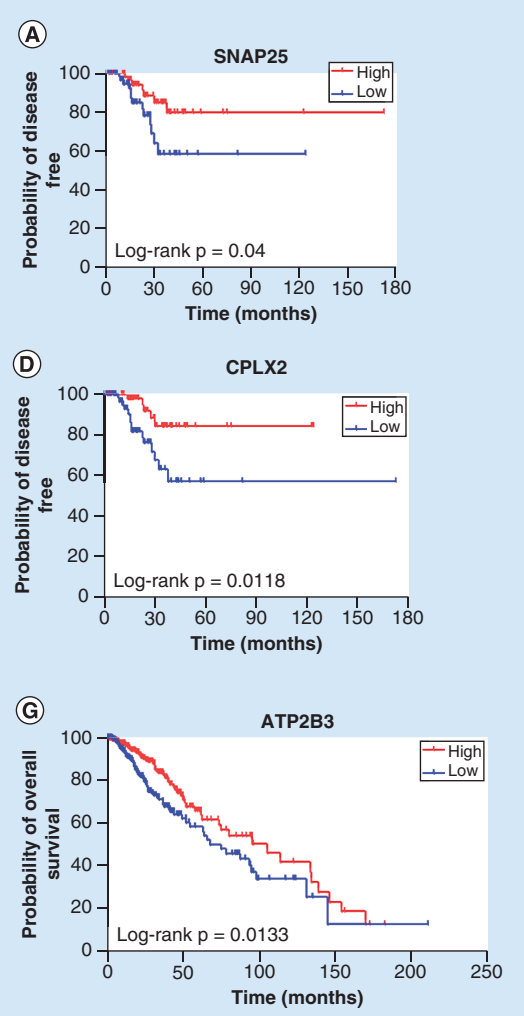

(J)

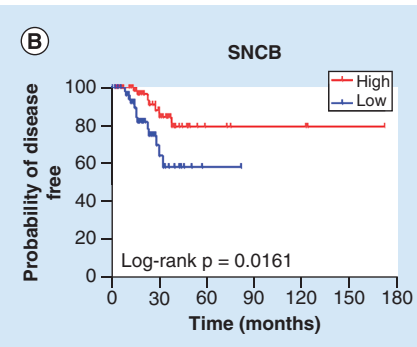

(E)
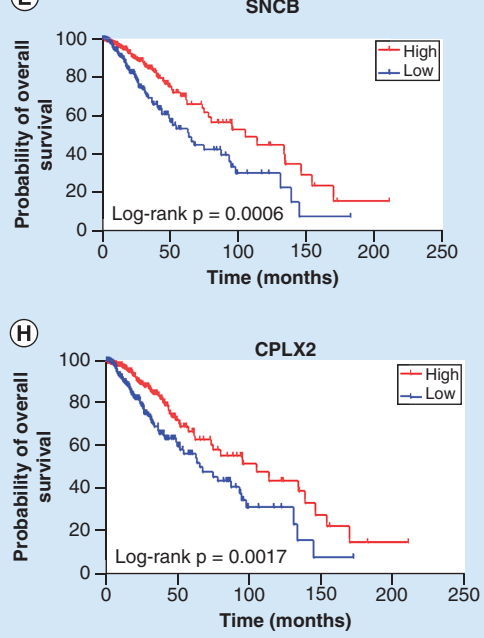

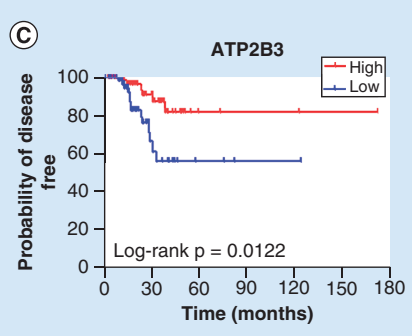

(F)

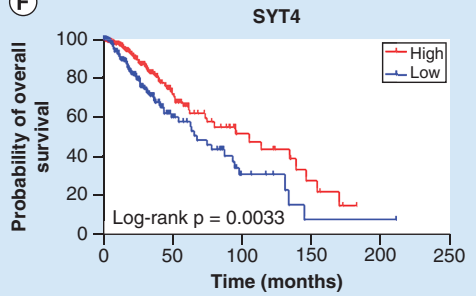

(1)

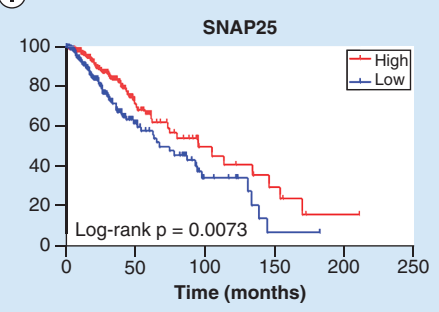

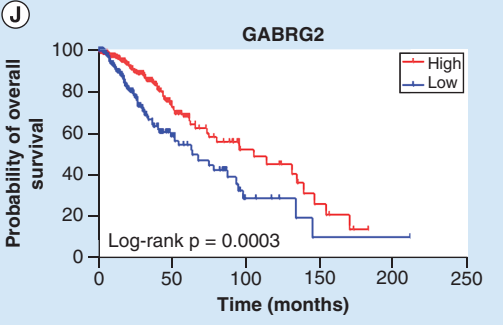

(K)

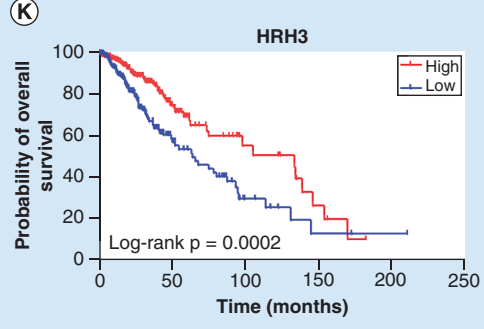

Figure 7. The prognosis analysis of the top hub genes in lower grade glioma. (A-C) The correlation of expression levels of SNAP25 (A), SNCB (B), ATP2BC (C) and CPLX2 (H) with disease-free survival. Log-rank test was used for analysis and a p-value $<0.05$ for statistical significance. (D-J) The correlation of expression levels of SNCB (E) and SYT4 (F), ATP2BC (G), CPLX2 (H), SNAP25 (I), GABRG2 (J) and HRH3 (K) with overall survival.

Log-rank test was used for analysis and a p-value $<0.05$ for statistical significance.

\section{Conclusion}

In summary, our research found the novel clinical significance of a TP53 mutation in LRGG and the associated critical genes and pathways. These results may help clinicians in identifying a better prognosis and planning more effective treatment strategies for patients.

\section{Supplementary data}

To view the supplementary data that accompany this paper please visit the journal website at: www.futuremedicine.com/doi/sup $\mathrm{pl} / 10.2217 / \mathrm{bmm}-2020-0331$

Financial \& competing interests disclosure

This work was supported by the grant from the Shanghai Municipal Commission of Health and Family Planning Research Project of China (no. 20164Y0170), the Fundamental Research Funds for the Central Universities (no. 22120180394), a grant from the Tenth People's Hospital of Tongji University of China Climbing Talented Person Plan (04.01.16.025) and the National Natural Science Foundation of China (no. 81901992). The authors declared no potential conflicts of interest with respect to the authorship and/or 
publication of this article. The authors have no other relevant affiliations or financial involvement with any organization or entity with a financial interest in or financial conflict with the subject matter or materials discussed in the manuscript apart from those disclosed.

No writing assistance was utilized in the production of this manuscript.

Ethical conduct of research

This study was approved by the Research Ethics Committee of Shanghai Tenth People's Hospital, PR China and they have followed the principles outlined in the Declaration of Helsinki for all human or animal experimental investigations.

\section{Open access}

This work is licensed under the Attribution-NonCommercial-NoDerivatives 4.0 Unported License. To view a copy of this license, visit http://creativecommons.org/licenses/by-nc-nd/4.0/

\section{Summary points}

- A TP53 mutant is the most frequently mutated gene in glioma, making it an important molecular biomarker for individualized therapeutic perspectives.

- The latest Cancer Genome Atlas data showed that a TP53 mutation was present in $49 \%$ of all lower grade (World Health Organization [WHO] grades II and III) glioma patients. Data from Genomics of Drug Sensitivity in Cancer database showed that three drugs: (5Z)-7-oxozeaenol, dabrafenib and nutlin-3a (-), were more resistance to patients with a TP53 mutation.

- A total of 1100 differentially expressed genes were identified and we did functional enrichment analysis. All the results showed that these genes are mainly concentrated in the transport of ionic and cancer-related pathways.

- The top ten hub genes were identified. The outcome analysis revealed that CPLX2, SNAP25, SNCB and ATP2B3 were significantly correlated with the disease-free survival rate and that the expression levels of SNAP25, SNCB, SYT4, ATP2B3, CPLX2, GABRG2 and HRH3 were significantly correlated with the overall survival rate.

- Our results identified the key genes and pathways, which might provide some basic evidence to improve glioma individualized treatment.

\section{References}

Papers of special note have been highlighted as: $\bullet \bullet$ of considerable interest

1. Grimm S, Chamberlain MC. Adult primary spinal cord tumors. Expert Rev. Neurother. 9(10), 1487-1495 (2009).

2. Taricco MA, Guirado VM, Fontes RB, Plese JP. Surgical treatment of primary intramedullary spinal cord tumors in adult patients. Arq. Neuropsiquiatr. 66(1), 59-63 (2008).

3. Brat DJ, Verhaak RG, Aldape KD et al. Comprehensive, integrative genomic analysis of diffuse lower-grade gliomas. N. Engl. J. Med. 372(26), 2481-2498 (2015).

-. Indicates the primary mutation types in lower grade glioma.

4. Louis DN, Ohgaki H, Wiestler OD et al. The 2007 WHO classification of tumours of the central nervous system. Acta Neuropathol. 114(2), 97-109 (2007).

5. Rogers TW, Toor G, Drummond K et al. The 2016 revision of the WHO classification of central nervous system tumours: retrospective application to a cohort of diffuse gliomas. J. Neurooncol. 137(1), 181-189 (2018).

-. Explains the new World Health Organization (WHO) classification of glioma.

6. Chamberlain MC, Tredway TL. Adult primary intradural spinal cord tumors: a review. Curr. Neurol. Neurosci. Rep. 11(3), 320-328 (2011).

7. Gibson GE, O'Grady A, Kay EW, Leader M, Murphy GM. p53 tumor suppressor gene protein expression in premalignant and malignant skin lesions of kidney transplant recipients. J. Am. Acad. Dermatol. 36(6 Pt 1), 924-931 (1997).

8. Vousden KH, Prives C. Blinded by the light: the growing complexity of p53. Cell 137(3), 413-431 (2009).

-. Shows the characteristics of tumor protein 53 comprehensively.

9. Taylor AM, Shih J, Ha G et al. Genomic and functional approaches to understanding cancer aneuploidy. Cancer Cell 33(4), 676-689 e673 (2018).

10. Liu J, Lichtenberg T, Hoadley KA et al. An integrated TCGA pan-cancer clinical data resource to drive high-quality survival outcome analytics. Cell 173(2), 400-416 (2018).

11. Lang GA, Iwakuma T, Suh YA et al. Gain of function of a p53 hot spot mutation in a mouse model of Li-Fraumeni syndrome. Cell 119(6), 861-872 (2004). 
12. Kim MP, Zhang Y, Lozano G. Mutant p53: Multiple mechanisms define biologic activity in cancer. Front. Oncol. 5, 249 (2015).

13. Morton JP, Timpson P, Karim SA et al. Mutant $\mathrm{p} 53$ drives metastasis and overcomes growth arrest/senescence in pancreatic cancer. Proc. Natl Acad. Sci. USA 107(1), 246-251 (2010).

14. Cerami E, Gao J, Dogrusoz U et al. The cBio cancer genomics portal: an open platform for exploring multidimensional cancer genomics data. Cancer Discov. 2(5), 401-404 (2012).

15. van den Heuvel JP, Maddox E, Maalouf SW et al. Replication study: systematic identification of genomic markers of drug sensitivity in cancer cells. eLife 7 (2018)

16. Yang W, Soares J, Greninger P et al. Genomics of drug sensitivity in cancer (GDSC): a resource for therapeutic biomarker discovery in cancer cells. Nucleic Acids Res. 41(Database issue), D955-D961 (2013).

17. Robinson MD, Mccarthy DJ, Smyth GK. edgeR: a bioconductor package for differential expression analysis of digital gene expression data. Bioinformatics 26(1), 139-140 (2010).

18. Mccarthy DJ, Chen Y, Smyth GK. Differential expression analysis of multifactor RNA-Seq experiments with respect to biological variation. Nucleic Acids Res. 40(10), 4288-4297 (2012).

19. Dennis G Jr, Sherman BT, Hosack DA et al. DAVID: database for annotation, visualization, and integrated discovery. Genome Biol. 4(5), P3 (2003).

20. Szklarczyk D, Franceschini A, Wyder $S$ et al. STRING v10: protein-protein interaction networks, integrated over the tree of life. Nucleic Acids Res. 43(Database issue), D447-D452 (2015).

21. Bader GD, Hogue CW. An automated method for finding molecular complexes in large protein interaction networks. $B M C$ Bioinformatics 4, 2 (2003).

22. Huang R, Liao X, Li Q. Identification of key pathways and genes in TP53 mutation acute myeloid leukemia: evidence from bioinformatics analysis. OncoTargets Ther. 11, 163-173 (2018).

23. Meek DW. The p53 response to DNA damage. DNA Repair (Amst.) 3(8-9), 1049-1056 (2004).

24. Wang XW, Harris CC. TP53 tumour suppressor gene: clues to molecular carcinogenesis and cancer therapy. Cancer Surv. 28, 169-196 (1996).

25. Ko $\mathrm{YH}$, Domingo-Vidal M, Roche $\mathrm{M}$ et al. TP53-inducible glycolysis and apoptosis regulator (TIGAR) metabolically reprograms carcinoma and stromal cells in breast cancer. J. Biol. Chem. 291(51), 26291-26303 (2016).

26. Zhang D, Yan H, Li H et al. TGFbeta-activated kinase 1 (TAK1) inhibition by 5 Z-7-oxozeaenol attenuates early brain injury after experimental subarachnoid hemorrhage. J. Biol. Chem. 290(32), 19900-19909 (2015).

27. Lu L, Zhang X, Tong H, Zhang W, Xu P, Qu S. Central administration of 5Z-7-oxozeaenol protects experimental autoimmune encephalomyelitis mice by inhibiting microglia activation. Front. Pharmacol. 8, 789 (2017).

28. Zhang D, Hu Y, Sun Q et al. Inhibition of transforming growth factor beta-activated kinase 1 confers neuroprotection after traumatic brain injury in rats. Neuroscience 238, 209-217 (2013).

29. Hauschild A, Grob JJ, Demidov LV et al. Dabrafenib in BRAF-mutated metastatic melanoma: a multicentre, open-label, Phase III randomised controlled trial. Lancet 380(9839), 358-365 (2012).

30. Brown NF, Carter T, Mulholland P. Dabrafenib in BRAFV600-mutated anaplastic pleomorphic xanthoastrocytoma. CNS Oncol. 6(1), 5-9 (2017)

31. Johanns TM, Ferguson CJ, Grierson PM, Dahiya S, Ansstas G. Rapid clinical and radiographic response with combined dabrafenib and trametinib in adults with BRAF-mutated high-grade glioma. J. Natl Compr. Canc. Netw. 16(1), 4-10 (2018).

32. Brown NF, Carter T, Kitchen N, Mulholland P. Dabrafenib and trametinib in BRAFV600E mutated glioma. CNS Oncol. 6(4), 291-296 (2017).

33. Janouskova $\mathrm{H}$, Maglott A, Leger DY et al. Integrin alpha5beta1 plays a critical role in resistance to temozolomide by interfering with the p53 pathway in high-grade glioma. Cancer Res. 72(14), 3463-3470 (2012).

34. Jiang L, Zhou J, Zhong D et al. Overexpression of SMC4 activates TGFbeta/Smad signaling and promotes aggressive phenotype in glioma cells. Oncogenesis 6(3), e301 (2017).

35. Gu Y, Cai R, Zhang C et al. miR-132-3p boosts caveolae-mediated transcellular transport in glioma endothelial cells by targeting PTEN/PI3K/PKB/Src/Cav-1 signaling pathway. FASEB J. 33(1), 441-454 (2019).

36. Kobayashi M, Mizutani A, Nishi $\mathrm{K}$ et al. Differences in accumulation and the transport mechanism of $\mathrm{l}$ - and d-methionine in high- and low-grade human glioma cells. Nucl. Med. Biol. 44, 78-82 (2017).

37. Pal J. Elucidating deregulated novel pathways in glioma through genetic and epigenetic approachese [PhD thesis]. Indian Institute of Science, Bangalore, India (2019).

38. Fung NH, Grima CA, Widodo SS et al. Understanding and exploiting cell signalling convergence nodes and pathway cross-talk in malignant brain cancer. Cell Signal. 57, 2-9 (2019). 
39. Park WJ, Lee SE, Kwon NS, Baek KJ, Kim DS, Yun HY. Leucine-rich glioma inactivated 3 associates with syntaxin 1. Neurosci. Lett. 444(3), 240-244 (2008).

40. Mason RA, Morlock EV, Karagas MR et al. EGFR pathway polymorphisms and bladder cancer susceptibility and prognosis. Carcinogenesis 30(7), 1155-1160 (2009). 